\title{
A Face Image Database for Evaluating Out-of-Focus Blur
}

\author{
Qi Han, Qiong Li and Xiamu Niu \\ Harbin Institute of Technology \\ China
}

\section{Introduction}

Face recognition is one of the most popular research fields of computer vision and machine learning(Tores (2004); Zhao et al. (2003)). Along with investigation of face recognition algorithms and systems, many face image databases have been collected(Gross (2005)). Face databases are important for the advancement of the research field. Because of the nonrigidity and complex 3D structure of face, many factors influence the performance of face detection and recognition algorithms such as pose, expression, age, brightness, contrast, noise, blur and etc. Some early face databases gathered under strictly controlled environment(Belhumeur et al. (1997); Samaria \& Harter (1994); Turk \& Pentland (1991)) only allow slight expression variation. To investigate the relationships between algorithms' performance and the above factors, more face databases with larger scale and various characters were built in the past years(Bailly-Bailliere et al. (2003); Flynn et al. (2003); Gao et al. (2008); Georghiades et al. (2001); Hallinan (1995); Phillips et al. (2000); Sim et al. (2003)). For instance, The "CAS-PEAL", "FERET", "CMU PIE", and "Yale B" databases include various poses(Gao et al. (2008); Georghiades et al. (2001); Phillips et al. (2000); Sim et al. (2003)); The "Harvard RL", "CMU PIE" and "Yale B" databases involve more than 40 different conditions in illumination(Georghiades et al. (2001); Hallinan (1995); Sim et al. (2003)); And the "BANCA", and "ND HID" databases contain over 10 times gathering(Bailly-Bailliere et al. (2003); Flynn et al. (2003)). These databases help researchers to evaluate and improve their algorithms about face detection, recognition, and other purposes.

Blur is not the most important but still a notable factor affecting the performance of a biometric system(Fronthaler et al. (2006); Zamani et al. (2007)). The main reasons leading blur consist in out-of-focus of camera and motion of object, and the out-of-focus blur is more significant in the application environment of face recognition(Eskicioglu \& Fisher (1995); Kim et al. (1998); Tanaka et al. (2007); Yitzhaky \& Kopeika (1996)). To investigate the influence of blur on a face recognition system, a face image database with different conditions of clarity and efficient blur evaluating algorithms are needed. This chapter introduces a new face database built for the purpose of blur evaluation. The application environments of face recognition are analyzed firstly, then a image gathering scheme is designed. Two typical gathering facilities are used and the focus status are divided into 11 steps. Further, the blur assessment algorithms are summarized and the comparison between them is raised on the various-clarity database. The 
result of the comparison may give some advice on the selection of blur assessment algorithms and onwards help controlling the quality of the face recognition system.

The rest of this chapter is organized as follow: section 2 introduces the gathering scheme of the database and part of the reason. Section 3 describes the content of the database. Section 4 summarizes the blur assessment algorithms and raises test and comparison of them. The results are also shown in this section. Finally, the conclusion and future works are given in section 5 .

\section{The acquiring scheme of database}

Most of the blur of digital image is caused by out-of-focus of camera or motion of objects. In the environments of face recognition system, the motion of face is not so fast, and a faster shutter may eliminate the motion blur in this case. Hence, the blur of face image is mostly caused by the out-of-focus of camera. The purpose of the new database is to help evaluating the blur assessment algorithms used in face recognition systems and the effect of the blur image on face recognition algorithms.

Around the topic of the database, an acquiring scheme is designed. The characters of the acquiring equipments are shown in table 1 .

\begin{tabular}{|c|c|c|}
\hline Equipment & PC camera & Professional camera \\
\hline Number of pixels & $1,300,000$ & $10,200,000$ \\
\hline Focus mode & manually & manually \\
\hline Focus states & 11 & 11 \\
\hline
\end{tabular}

Table 1. Characters of acquiring equipments

28 volunteers were invited to contribute. The focus states are divided into 11 step include 10 out-of-focus states and 1 correct focused state. The expression is not restricted. Wearing glasses is allowed but will be gathered twice with glasses and without glasses. So the two equipments is expected to gather 682 frontal facial images in total.

\section{The contents of database}

The contents of the database are described in table 2. The database contains two parts of face images gathered from two typical facilities - a PC camera (Part I) and a digital single-lens reflex (DSLR) camera (Part II). Images in part I usually come with the face recognition applications in PC, mobile phone, ineternet authentication, and etc. While images in part II appear more in face detection, face retrieval and some other applications.

Figure 1 and 2 show some example images in the blur database. Figure 1 show images in part I and figure 2 show which in part II. 11 images of each faces are grouped into one troop. The correct focused image in part I is no.6, and that in part II is no.4. The shown samples are selected from the 11 images of each group(No.1,3,5,6,9,11 from part I, No.1,2,3,5,7,9 from part II). 


\begin{tabular}{|c|c|c|}
\hline & Part I & Part II \\
\hline Individuals & 28 & 28 \\
\hline Faces & 31 & 31 \\
\hline With glasses & 3 & 3 \\
\hline Male & 11 & 11 \\
\hline Female & 17 & 17 \\
\hline Race & Oriental & Oriental \\
\hline Pose & Frontal & Frontal \\
\hline Correct focus & 1 image & 1 image \\
\hline Out of focus & 10 images & 10 images \\
\hline Correct focused & No.6 & No.4 \\
\hline Image size & $320 \times 240$ & $2896 \times 1936$ \\
\hline
\end{tabular}

Table 2. Overview of the blur face image databases

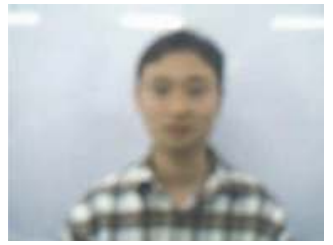

(a) Image No.1

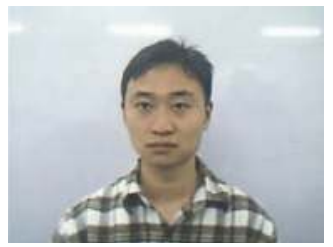

(d) Image No.6

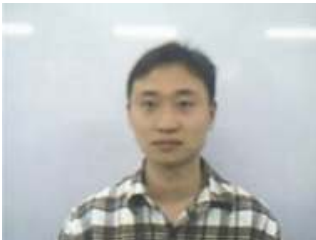

(b) Image No.3

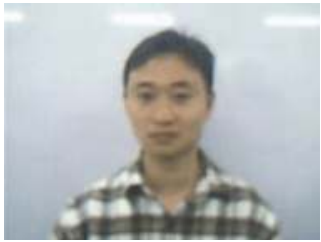

(e) Image No.9

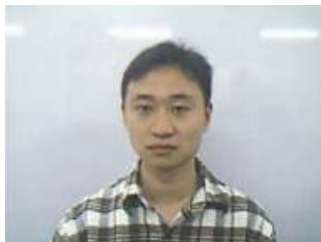

(c) Image No.5

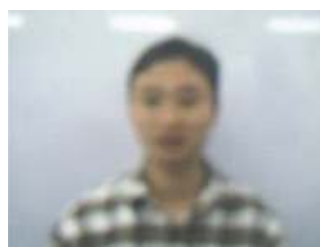

(f) Image No.11

Fig. 1. Images acquired by PC camera (Fig.1(d) is the correct focused image)

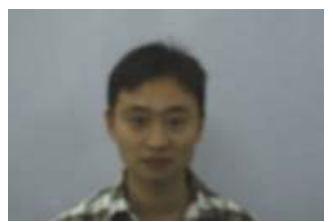

(a) Image No.1

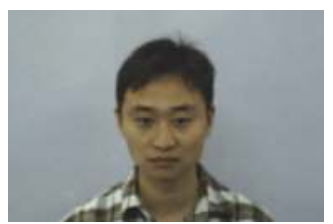

(d) Image No.5

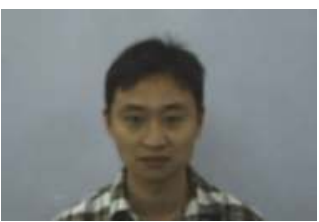

(b) Image No.2

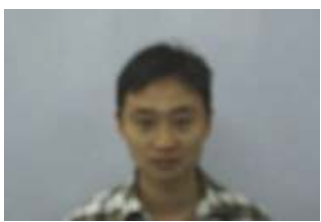

(e) Image No.7

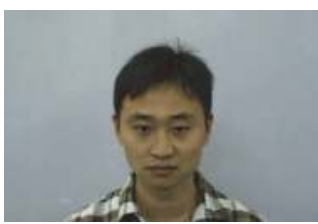

(c) Image No.4

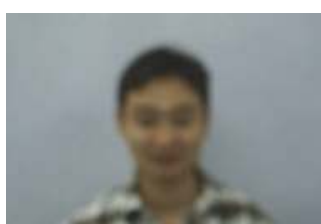

(f) Image No.9

Fig. 2. Images acquired by DSLR camera (Fig.6(c) is the correct focused image) 


\section{The evaluation on blur assessment algorithms}

Based on the database aiming on the image character of out-of-focus blur, a comprehensive evaluation of blur assessment algorithms is raised. The more credible benchmarking database, the result may be more universal - at least in the application fields related with face image.

\subsection{The blur assessment algorithms}

There are a great deal of blur assessment algorithms in literatures(Kim \& Paik (1998); Li (2004); Maoyong et al. (2001); Subbarao et al. (1993)). Typical algorithms are summarized as follow (Suppose $f(x, y)$ is the grey scale value of pixel $(x, y)$ of the image, $N_{x}, N_{y}$ are the size of the image, and $s$ is the clarity score.):

1. Variation of grey scale based algorithms:

(alg-1) Variance of grey scale:

$$
\begin{aligned}
& \bar{g}=\frac{1}{N_{x} \times N_{y}} \sum_{x=1}^{N_{x}} \sum_{y=1}^{N_{y}} f(x, y) \\
& s=\frac{1}{N_{x} \times N_{y}} \sum_{x=1}^{N_{x}} \sum_{y=1}^{N_{y}}(f(x, y)-\bar{g})^{2}
\end{aligned}
$$

(alg-2) Grey scale threshold. Define the average grey scale of a image as $u$. $f_{H}(x, y)$ means the grey scale value of all the $C_{H}$ pixels satisfying $f(x, y)>(1+W) u$, and $f_{L}(x, y)$ means those of $C_{L}$ pixels satisfying $f(x, y)<(1+W) u$. Then the clarity score is computed as:

$$
\begin{aligned}
& \left\{\begin{array}{l}
H=\sum_{x=1}^{N_{x}} \sum_{y=1}^{N_{y}} f_{H}(x, y) / C_{H} \\
L=\sum_{x=1}^{N_{x}} \sum_{y=1}^{N_{y}} f_{L}(x, y) / C_{L}
\end{array}\right. \\
& S=(H-L) /(H+L)
\end{aligned}
$$

2. Gradient based algorithms:

(alg-3) Sum of modulus of grey scale difference:

$$
\begin{aligned}
& \Delta_{x} f(x, y)=f(x+1, y)-f(x, y) \\
& \Delta_{y} f(x, y)=f(x, y+1)-f(x, y) \\
& s=\sum_{x=1}^{N_{x}-1} \sum_{y=1}^{N_{y}-1}\left\{\left|\Delta_{x} f(x, y)\right|-\left|\Delta_{y} f(x, y)\right|\right\}
\end{aligned}
$$

(alg-4) Brenner function:

$$
s=\sum_{x=1}^{N_{x}-2} \sum_{y=1}^{N_{y}}(f(x+2, y)-f(x, y))^{2}
$$


(alg-5) Square plane sum modulus difference:

$$
S=\sum_{x=1}^{N_{x}-1} \sum_{y=1}^{N_{y}-1}\left(\Delta_{x}^{2}+\Delta_{y}^{2}\right)
$$

(alg-6) Sum of Roberts gradient:

$$
\begin{aligned}
\mathcal{S}= & \sum_{x=1}^{N_{x}-1} \sum_{y=1}^{N_{y}-1}\{|f(x, y)-f(x+1, y+1)| \\
& +|f(x+1, y)-f(x, y+1)|\}
\end{aligned}
$$

(alg-7) Sum of Laplace gradient I:

$$
\begin{aligned}
& \nabla f(x, y)=[f(x+1, y)+f(x-1, y) \\
& +f(x, y+1)+f(x, y-1)]-4 f(x, y) \\
& s=\sum_{x=2}^{N_{x}-1} \sum_{y=2}^{N_{y}-1}|\nabla f(x, y)|
\end{aligned}
$$

(alg-8) Sum of Laplace gradient II:

$$
\begin{aligned}
& \nabla f(x, y)=[f(x+1, y)+f(x-1, y) \\
& +f(x, y+1)+f(x, y-1)+f(x+1, y-1) \\
& +f(x-1, y+1)+f(x+1, y-1) \\
& +f(x-1, y-1)]-8 f(x, y) \\
& s=\sum_{x=2}^{N_{x}-1} \sum_{y=2}^{N_{y}-1}|\nabla f(x, y)|
\end{aligned}
$$

(alg-9) Tenengrad function, define $f_{x}(x, y)=f(x, y) * K_{x}$ and $f_{y}(x, y)=f(x, y) * K_{y}$, where:

$$
K_{x}=\left[\begin{array}{rrr}
0 & -1 & 0 \\
0 & 2 & 0 \\
0 & -1 & 0
\end{array}\right], K_{y}=\left[\begin{array}{ccc}
0 & 0 & 0 \\
-1 & 2 & -1 \\
0 & 0 & 0
\end{array}\right]
$$

Then:

$$
s=\sqrt{f_{x}^{2}(x, y)+f_{y}^{2}(x, y)}
$$

3. (alg-10) Grey scale entropy based algorithm, define $p(g)$ as the probability of the grey scale value $g$ satisfies $g \in[0, G]$. Then:

$$
s=-\sum_{g=0}^{G} p(g) \log p(g)
$$


4. (alg-11) Fourier-Transform based algorithm:

$$
\begin{aligned}
& F(u, v)=\sum_{x=1}^{N_{x}} \sum_{x=1}^{N_{y}} f(x, y) e^{\left[-j 2 \pi\left(x u / N_{x}+y v / N_{y}\right)\right]} \\
& s=\left[\operatorname{Re}\left(\frac{F(u, v)}{\sum_{x=1}^{N} \sum_{y=1}^{N_{y}} f(x, y)}\right)\right]^{2}+\left[\operatorname{Im}\left(\frac{F(u, v)}{\sum_{x=1}^{N_{x}} \sum_{y=1}^{N_{y}} f(x, y)}\right)\right]^{2}
\end{aligned}
$$

5. (alg-12) Wavelet-Transform based algorithm. Suppose $A$ is the vector of decompose coefficients, the image size is $l \times l$, then:

$$
s=\frac{1}{l^{2}-1}\left[\sum_{i=\left(l / 2^{m}\right)^{2}}^{l^{2}-1} A[i]^{2}\right]
$$

6. (alg-13) Median filtering based algorithm. Define $F^{\prime}$ is the $3 \times 3$ median filtered result of image $F . D=\left|F-F^{\prime}\right|$, then:

$$
s=\sum_{x} \sum_{y} D(x, y)
$$

Alg-1 to alg-13 are the most frequently used algorithms in literatures which cover the spatial domain and frequency domain methods. The evaluation over them aims to investigate their performance on face images. The references will be the clarity information recorded at gathering of the database. The guideline to evaluate an algorithm is correctness, susceptiveness and unimodality of the curve on the testing image database.

\subsection{The evaluation on the database}

To evaluate the performance of the blur assessment algorithms effectively, an evaluation scheme is designed as follow:

1. Implement a blur assessment algorithm $A_{i}$;

2. Compute the clarity scores of each image in each group, and get $31 \times 11 \times 2$ scores of the $i$ th algorithm: $\left(s_{1, i}^{I}, s_{2, i}^{I}, \cdots, s_{11, i}^{I}\right), \cdots,\left(s_{1, i}^{I I}, s_{2, i}^{I I}, \cdots, s_{11, i}^{I I}\right)$;

3. Compute the average scores of each group in each part, and get 2 score sequences of each part of the database: $\left(s_{1}^{I}, s_{2}^{I}, \cdots, s_{11}^{I}\right)$ and $\left(s_{1}^{I I}, s_{2}^{I I}, \cdots, s_{11}^{I I}\right)$;

4. Go to step 1, repeat the process until all algorithms get their scores;

5. Plot these scores and analysis the result.

Because the image size of part II is $2896 \times 1936$ which is much bigger than that in part I $(320 \times 240)$. Another data set named part III is acquired via sampling from image in part II. The image size of part III is $362 \times 242$ which is close to part I. Some interesting results will be shown in the next section.

\subsection{The results and analysis}

The results of the evaluation are shown in figure 3 to figure 5. The algorithm-9 exhibits the best performance in part I and part III of database, while the algorithm- 12 shows the stablest 


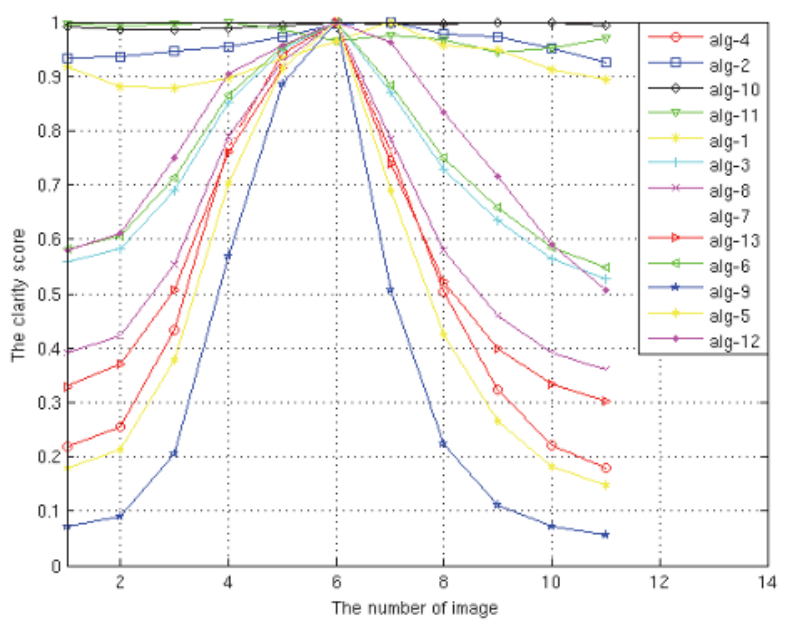

Fig. 3. The evaluating curves on part I

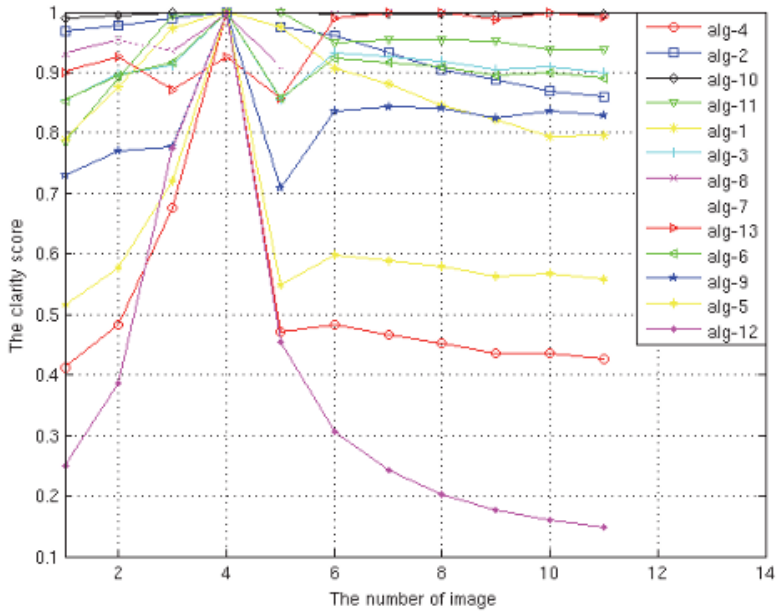

Fig. 4. The evaluating curves on part II 


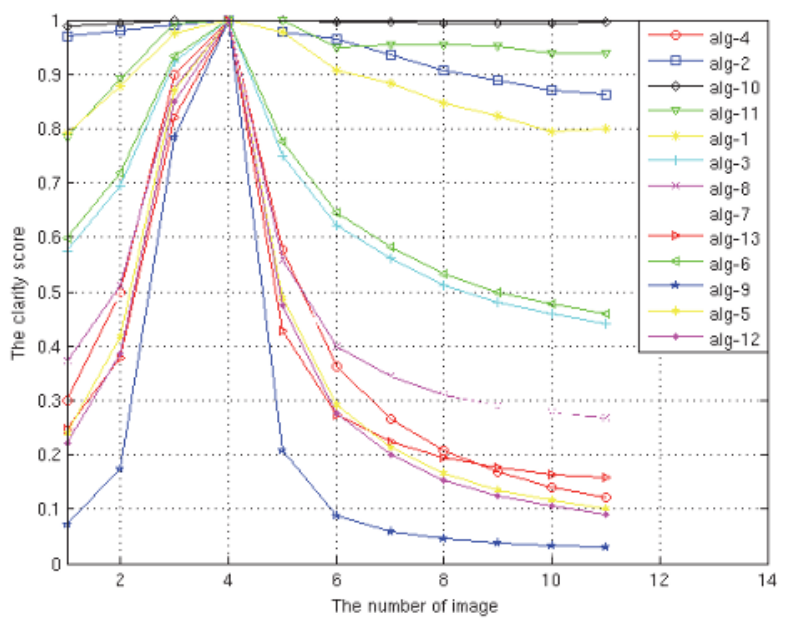

Fig. 5. The evaluating curves on part III

performance in the three testing sets even though it's curves are not the steepest curves in part I and III.

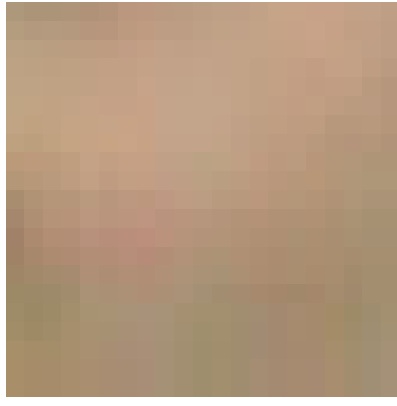

(a) $21 \times 21$ block from part I

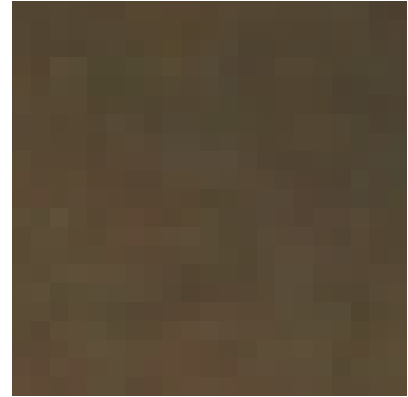

(b) $21 \times 21$ block from part II

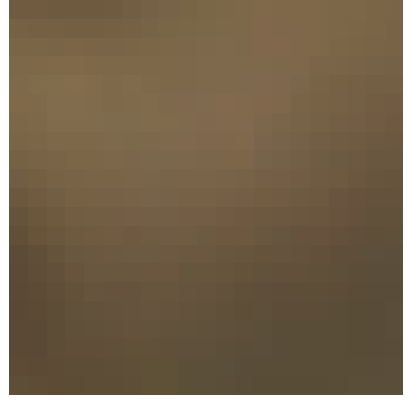

(c) $21 \times 21$ block from part III

Fig. 6. Comparison of small pixel blocks from different part of database

And there is an interesting phenomenon shown by the curves: most of the blur assessment algorithms give the not so bad image lower scores in database part II. These curves lost their unimodality in this case. In fact, this result reveals an important guide line of blur assessing on images of different sizes: To small size images such as images taken by PC camera, most of the current blur assessment algorithms work well; but only a few algorithms keep their performance on big size images. The reason may be explained partly by figure 6 . The three images are the amplified pixel blocks in a image. Their real size is $21 \times 21$ pixels. They are cut separately from a smooth area of blur images of each part. The noise from camera makes notable spots whose size is comparable with the size of many algorithms' mask. So only the algorithms which use the global features won't be influence too much by these spots. In that way the curves of algorithm 1,2, 11 and 12 could keep their unimodality. So the 
evaluation suggests that the choosing of blur assessment algorithm should depend on the images' features, especially the size and noise spots' influence. The figure 3 to 5 could serve as a reference to help choosing the suitable algorithm.

\section{Conclusion and future works}

Out-of-focus blur is an important factor related with the performance of face detection, recognition and retrieval algorithms. This chapter describes a new database specially aiming at the factor of out-of-focus blur. And a comprehensive evaluation of blur assessment algorithms is raised which takes this database as a benchmark. 13 typical algorithms are evaluated on three datasets with different features. Some useful results are revealed from the result which may be a guideline for choosing suitable algorithms in a certain application. The future works may include further evaluation of face detection and recognition algorithms, and the applications of the guidelines in a real system.

\section{References}

Bailly-Bailliere, E., Bengio, S., Bimbot, F., Hamouz, M., Kittler, J., J.Mariethoz, J.Matas, K.Messer, Popovici, V., Poree, F., Ruiz, B. \& Thiran, J.-P. (2003). The banca database and evaluation protocol, Proceedings of Audio- and Video-Based Biometric Person Authentication (AVBPA), pp. 625-638.

Belhumeur, P., Hespanha, J. \& Kriegman, D. (1997). Eigenfaces vs. fisherfaces: Recognition using class specific linear projection, IEEE Transactions on Pattern Analysis and Machine Intelligence 19(7): 711-720.

Eskicioglu, A. \& Fisher, P. (1995). Image quality measures and their performance, IEEE Transactions on Communications 43(12): 2959-2965.

Flynn, P., Bowyer, K. \& Phillips, P. J. (2003). Assesment of time dependency in face recognition: an initial study, Proceedings of Audio- and Video-Based Biometric Person Authentication (AVBPA), pp. 44-51.

Fronthaler, H., Kollreider, K. \& Bigun, J. (2006). Automatic image quality assessment with application in biometrics, Proceedings of the 2006 Conference on Computer Vision and Pattern Recognition Workshop (CVPRWaŕr06).

Gao, W., Cao, B., Shan, S., Chen, X., Zhou, D., Zhang, X. \& Zhao, D. (2008). The cas-peal large-scale chinese face database and baseline evaluations, IEEE Transactions on Systems, Man and Cybernetics, Part A 38(1): 149-161.

Georghiades, A., Belhumeur, P. \& Kriegman, D. (2001). From few to many: illumination cone models for face recognition under variable lighting and pose, IEEE Transactions on Pattern Analysis and Machine Intelligence 23(6): 643-660.

Gross, R. (2005). Face databases, in A. S.Li (ed.), Handbook of Face Recognition, Springer, New York.

Hallinan, P. (1995). A Deformable Model for Face Recognition under Arbitrary Lighting Conditions, $\mathrm{PhD}$ thesis, Harvard University.

Kim, S. K. \& Paik, J. K. (1998). Out-of-focus blur estimation and restoration for digital auto-focusing system, Electronics Letters 34(12): 1217-1219. 
Kim, S. K., Park, S. R. \& Paik, J. K. (1998). Simultaneous out-of-focus blur estimation and restoration for digital auto-focusing system, IEEE Transactions on Consumer Electronics 44(3): 1071-1075.

Li, Q. (2004). Studies on the Theory and Implementation Method of Digital Autofocus Technology, PhD thesis, Zhejiang University.

Maoyong, C., Nongliang, S. \& Daoyin, Y. (2001). Study on clarity-evaluation-function of out-of-focus blurred image, Chinese Journal of Scientific Instrument 22(3): 261-264.

Phillips, P. J., Moon, H., Rizvi, S. A. \& Rauss, P. J. (2000). The feret evaluation methodology for face-recognition algorithms, IEEE Transactions on Pattern Analysis and Machine Intelligence 22(10): 1090-1104.

Samaria, F. \& Harter, A. (1994). Parameterisation of a stochastic model for human face identification, Proceedings of the Second IEEE Workshop on Applications of Computer Vision, pp. 138-142.

Sim, T., Baker, S. \& Bsat, M. (2003). The cmu pose, illumination, and expression database, IEEE Transactions on Pattern Analysis and Machine Intelligence 25(12): 1615-1618.

Subbarao, M., Choi, T. \& Nikzad, A. (1993). Focusing techniques, Optical Engineering 32(11): 2824-2836.

Tanaka, M., Yoneji, K. \& Okutomi, M. (2007). Motion blur parameter identification from a linearly blurred image, Proceedings of Digest of Technical Papers. International Conference on Consumer Electronics, pp. 1-2.

Tores, L. (2004). Is there any hope for face recognition?, Proceedings of the 5th Internationl Workshop on Image Analysis for Multimedia Interactive Services (WIAMIS '04), Lisbon, Portugal, pp. 2709-2712.

Turk, M. A. \& Pentland, A. P. (1991). Face recognition using eigenfaces, Proceedings of the IEEE Conference on Computer Vision and Pattern Recognition, IEEE, Hawaii, USA, pp. 586-591.

Yitzhaky, Y. \& Kopeika, N. (1996). Evaluation of the blur parameters from motion blurred images, Proceedings of the Nineteenth Convention of Electrical and Electronics Engineers in Israel, pp. 216-219.

Zamani, A. N., Awang, M. K., Omar, N. \& Nazeer, S. A. (2007). Image quality assessments and restoration for face detection and recognition system images, Proceeding of The 2nd National Intelligent Systems And Information Technology Symposium (ISITS'07).

Zhao, W., Chellappa, R., Phillips, P. J. \& Rosenfeld, A. (2003). Face recogintion: A literature survey, ACM Computing Surveys 35(4): 399-458. 


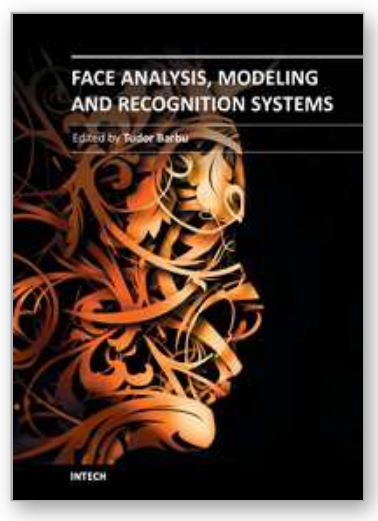

\author{
Face Analysis, Modeling and Recognition Systems \\ Edited by Dr. Tudor Barbu
}

ISBN 978-953-307-738-3

Hard cover, 212 pages

Publisher InTech

Published online 30, September, 2011

Published in print edition September, 2011

The purpose of this book, entitled Face Analysis, Modeling and Recognition Systems is to provide a concise and comprehensive coverage of artificial face recognition domain across four major areas of interest:

biometrics, robotics, image databases and cognitive models. Our book aims to provide the reader with current state-of-the-art in these domains. The book is composed of 12 chapters which are grouped in four sections. The chapters in this book describe numerous novel face analysis techniques and approach many unsolved issues. The authors who contributed to this book work as professors and researchers at important institutions across the globe, and are recognized experts in the scientific fields approached here. The topics in this book cover a wide range of issues related to face analysis and here are offered many solutions to open issues. We anticipate that this book will be of special interest to researchers and academics interested in computer vision, biometrics, image processing, pattern recognition and medical diagnosis.

\title{
How to reference
}

In order to correctly reference this scholarly work, feel free to copy and paste the following:

Qi Han, Qiong Li and Xiamu Niu (2011). A Face Image Database for Evaluating Out-of-Focus Blur, Face Analysis, Modeling and Recognition Systems, Dr. Tudor Barbu (Ed.), ISBN: 978-953-307-738-3, InTech, Available from: http://www.intechopen.com/books/face-analysis-modeling-and-recognition-systems/a-faceimage-database-for-evaluating-out-of-focus-blur

\section{INTECH}

open science | open minds

\section{InTech Europe}

University Campus STeP Ri

Slavka Krautzeka 83/A

51000 Rijeka, Croatia

Phone: +385 (51) 770447

Fax: +385 (51) 686166

www.intechopen.com

\section{InTech China}

Unit 405, Office Block, Hotel Equatorial Shanghai

No.65, Yan An Road (West), Shanghai, 200040, China

中国上海市延安西路65号上海国际贵都大饭店办公楼 405 单元

Phone: +86-21-62489820

Fax: $+86-21-62489821$ 
(C) 2011 The Author(s). Licensee IntechOpen. This chapter is distributed under the terms of the Creative Commons Attribution-NonCommercialShareAlike-3.0 License, which permits use, distribution and reproduction for non-commercial purposes, provided the original is properly cited and derivative works building on this content are distributed under the same license. 